\title{
3D Electron Ptychography
}

Si Gao ${ }^{1}$, Zhiyuan Ding ${ }^{1}$, Xiaoqing Pan $^{5}$, Angus. I. Kirkland ${ }^{2,3}$ and Peng Wang ${ }^{1}$

1. National Laboratory of Solid State Microstructures, College of Engineering and Applied Sciences and Collaborative Innovation Center of Advanced Microstructures, Nanjing University, Nanjing, People's Republic of China.

2. Department of Materials, University of Oxford, Parks Road, Oxford, UK.

3. Electron Physical Sciences Imaging Centre, Diamond Lightsource Ltd., Diamond House, U.K.

4. JEOL Ltd, 1-2 Mushashino, 3-Chome, Akishima, Tokyo, Japan.

5. Department of Chemical Engineering and Materials Science and Department of Physics and Astronomy, University of California, Irvine, CA, USA.

Since the development of aberration correctors and key electron optical components (i.e. high coherence electron soureces), scanning transmission electron microscopy (STEM) has become a widely used and powerful technique for materials science research. However, there still remain challenges in the use of this method in some areas, such as studies of beam sensitive materials and three-dimensional quantitative imaging. It has been shown that ptychography is a candidate for tackling these challenges due to its potential for super resolution [1,2], high phase sensitivity [3-5], low-dose [6] and threedimensional information recovery [4,7]. In addition, a new generation of fast direct electron detectors together with the stability of modern electron microscopes has enabled two dimensional (2D) ptychographic reconstructions from hundreds of thousands of probe positions [2,4,5]. However, the dwell time per probe position is still of the order of $\mathrm{ms}$ which is several orders of magnitude longer than that used for conventional ADF imaging. Using focused-probe ptychography, probe arrays equivalent to those used in conventional annular dark field imaging (ADF) imaging are required to satisfy the sampling requirements over a reasonable field of view, resulting in a relatively high cumulative dose at the sample and susceptibility to sample drift. Therefore, it becomes ever more challenging to extend this type of ptychography to the recording of an entire tilt series for tomographic reconstruction.

An alternate technique is defocused ptychography as shown in Fig. 1a), where a probe defocused by several tens of nm's is scanned over a specimen with highly overlapping probe positions. Using this technique only a few hundred probe positions are required to reconstruct the $2 \mathrm{D}$ phase. This has been successfully used in both imaging light atoms in $\mathrm{a} \mathrm{LaB}_{6}$ crystal [3] and $\mathrm{MoS}_{2}$ at low dose [6]. The detection characteristics and high speed of modern direct detectors combined with the defocused nature of the probe and the limited number of probe positions required offers the potential for $3 \mathrm{D}$ phase recovery.

In this work, we firstly demonstrate that defocused-probe ptychography using an inverse multislice method can produce 3D optically sectioned phase data from a thick sample as shown in Fig. 1 a). The experimental was carried out on a sample of carbon nanotube bundles (CNTs) as shown in Fig. 1 b) at $60 \mathrm{kV}$. Using the 3PIE algorithm [4], the phase of the sample at different sample heights as shown in Fig. $1 \mathrm{c})$ and d) were reconstructed. The method provides high contrast, quantitative phase maps at close to atomic lateral resolution and with a few tens of nanometers depth resolution. An additional advantage is that the capability for post-acquisition focusing provides data at an optimal defocus, which is potentially beneficial for imaging beam sensitive materials. We will also show some initial results of 3D ptychographic phase imaging on nanostructured materials using a tilt series based approach, where a 4D 
ptychographic data set is collected for a range of tilt angles for $2 \mathrm{D}$ phase reconstruction followed by tomographic reconstruction performed with different techniques, i.e. the BP and SIRT methods [9].

References:

[1] PD Nellist, BC McCallum and JM Rodenburg, Nature 374 (1995), p. 630.

[2] Y Jiang et al., Nature 559 (2018), p. 343.

[3] P Wang et al. Scientific Reports 7 (2017), p. 2857.

[4] H Yang et al., Nature Communications 7 (2016), p. 12532.

[5] B Song et al., Physical Review Letters 121 (2018), p. 146101.

[6] J Song et al., Scientific Reports, Accepted.

[7] S Gao et al., Nature Communications 8 (2017), p. 163.

[8] AM Maiden, MJ Humphry and JM Rodenburg, Journal of the Optical Society of America a 29 (2012), p. 1606.

[9] The authors acknowledge funding from the National Natural Science Foundation of China (11874199, 11474147), and the National Basic Research Program of China, (2015CB654901).

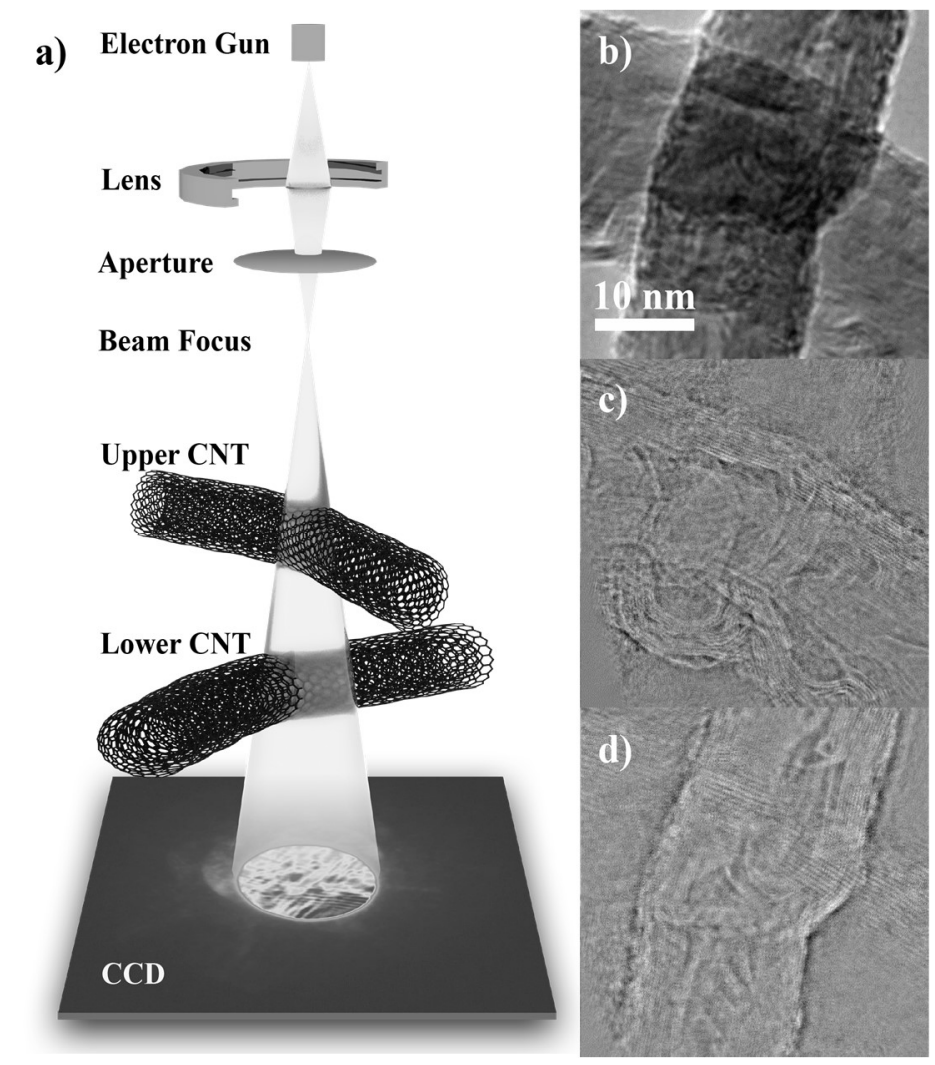

Figure 1. (a) Schematic of the experimental set-up for defocused probe ptychography; (b) TEM image of the CNTs; (c) and (d) Phase of the upper and lower CNTs at Gaussian focus, respectively. 